\title{
Time-Resolved Distance Determination by Tryptophan Fluorescence Quenching: Probing Intermediates in Membrane Protein Folding ${ }^{\dagger}$
}

\author{
Jörg H. Kleinschmidt and Lukas K. Tamm* \\ Department of Molecular Physiology and Biological Physics and Center for Structural Biology, University of Virginia Health \\ Sciences Center, P.O. Box 10011, Charlottesville, Virginia 22906-0011
}

Received October 15, 1998; Revised Manuscript Received January 26, 1999

\begin{abstract}
The mechanism of insertion and folding of an integral membrane protein has been investigated with the $\beta$-barrel forming outer membrane protein A (OmpA) of Escherichia coli. This work describes a new approach to this problem by combining structural information obtained from tryptophan fluorescence quenching at different depths in the lipid bilayer with the kinetics of the refolding process. Experiments carried out over a temperature range between 2 and $40{ }^{\circ} \mathrm{C}$ allowed us to detect, trap, and characterize previously unidentified folding intermediates on the pathway of OmpA insertion and folding into lipid bilayers. Three membrane-bound intermediates were found in which the average distances of the Trps were $14-16,10-11$, and $0-5 \AA$, respectively, from the bilayer center. The first folding intermediate is stable at $2{ }^{\circ} \mathrm{C}$ for at least $1 \mathrm{~h}$. A second intermediate has been isolated at temperatures between 7 and 20 ${ }^{\circ} \mathrm{C}$. The Trps move $4-5 \AA$ closer to the center of the bilayer at this stage. Subsequently, in an intermediate that is observable at $26-28{ }^{\circ} \mathrm{C}$, the Trps move another $5-10 \AA$ closer to the center of the bilayer. The final (native) structure is observed at higher temperatures of refolding. In this structure, the Trps are located on average about 9-10 $\AA$ from the bilayer center. Monitoring the evolution of Trp fluorescence quenching by a set of brominated lipids during refolding at various temperatures therefore allowed us to identify and characterize intermediate states in the folding process of an integral membrane protein.
\end{abstract}

Cells synthesize proteins as linear sequences of amino acids, which then fold into unique three-dimensional structures usually representing their lowest energy state. In dilute solution, protein folding can occur spontaneously and does not require external energy nor any cofactors. However, in the crowded environment within cells, molecular chaperone proteins are needed to prevent misfolding and to allow proteins to kinetically find their native state. Although most experimental work on protein folding has been carried out with water-soluble proteins, it is generally assumed that similar fundamental principles hold true for the folding of integral membrane proteins. Spontaneous refolding into lipid bilayers has been demonstrated for the outer membrane protein A (OmpA) ${ }^{1}$ of Escherichia coli $(1,2)$ and for fragments of bacteriorhodopsin (BR) $(3,4)$. There is strong evidence that OmpA forms an eight-stranded $\beta$-barrel in lipid bilayers (5-7). In contrast, BR consists of seven closely packed transmembrane $\alpha$-helices $(8,9)$ and therefore has often served as the structural prototype for seven-helix receptors. Although $\beta$-barrel structured membrane proteins

\footnotetext{
Supported by Grant GM51329 from the National Institutes of Health.

* Corresponding author. Telephone: 804-982-3578. Fax: 804-9821616. E-mail: 1kt2e@virginia.edu.

${ }_{1}^{1}$ Abbreviations: BR, bacteriorhodopsin; $m, n$-DiBrPC, 1-palmitoyl2-(m,n-dibromo)stearoyl-sn-glycero-3-phosphocholine; DOPC, 1,2-dioleoyl-sn-glycero-3-phosphocholine; EDTA, ethylenediaminetetraacetic acid; OmpA, outer membrane protein A; PAGE, polyacrylamide gel electrophoresis; SDS, sodium dodecyl sulfate; SUV, small unilamellar vesicle; TDFQ, time-resolved distance determination by fluorescence quenching; Trp, tryptophan.
}

are most likely less abundant than helical membrane proteins, $\beta$-barrels offer distinct advantages for folding studies: they become completely unfolded in urea or guanidinium chloride (10), and they spontaneously refold into detergent micelles or preformed lipid bilayers (without detergent) upon dilution of the denaturant $(1,11-13)$. Utilizing these denaturing/ refolding techniques, we have previously discovered two membrane-bound intermediates in the refolding process of OmpA (2). The experimental tools of that previous study were an SDS-polyacrylamide gel-shift assay and observations of changes of intrinsic tryptophan fluorescence as a function of time at different temperatures. Completely in vitro folded OmpA is indistinguishable from native OmpA as judged by numerous spectroscopic (CD, FTIR, Raman, fluorescence), biochemical (protease accessibility, mobility on native SDS gels), and immunological (exposure of characteristic epitopes) criteria. Also, refolded OmpA forms ion channels in planar lipid bilayers as does native OmpA (Arora and Tamm, unpublished results).

Steady-state and time-resolved tryptophan fluorescence experiments have significantly contributed to our present understanding of the folding of water-soluble proteins. Collisional quenchers of tryptophan fluorescence have also been used frequently to characterize folded and unfolded states of proteins. For example, the accessibility of tryptophans, and thus their degree of exposure to solvent, has been probed by polar (e.g., cesium, iodide, acrylamide) or apolar (e.g., molecular oxygen) quenchers (14). The concept of probing the microenvironment of proteins has been extended to membrane proteins by using a set of lipids with 
covalently attached quencher groups in different positions along the $s n-2$ acyl chain in a lipid bilayer. Nitroxide- or bromine-labeled fatty acids and phospholipids have been used to estimate the position of tryptophans of membrane proteins in the lipid bilayer $(15,16)$. This approach has become particularly useful with selectively brominated lipids because the positions and positional distributions of the bromines in the bilayer are known from X-ray diffraction $(17,18)$.

In the present work, we utilize the position-dependent quenching by selectively brominated lipids to probe the movement of the five Trp residues of OmpA during refolding and insertion into the lipid bilayer. In earlier work, we have shown that the kinetics of OmpA refolding in membranes are strongly temperature-dependent (2). These kinetics suggested that at temperatures $<10{ }^{\circ} \mathrm{C}$ at least one process is so slow that it should be possible to characterize an intermediate state by locating the Trps with a set of membrane-bound quenchers at different depths in the lipid bilayer. Therefore, we have developed a time-resolved fluorescence quenching technique that monitors Trp movement across the lipid bilayer at temperatures ranging from 2 to $40{ }^{\circ} \mathrm{C}$. We show that the vectorial transport of tryptophan residues across membranes can be followed by this method. In the accompanying paper we apply these techniques to the folding and membrane insertion of single tryptophan mutants of OmpA (19).

\section{MATERIALS AND METHODS}

Materials. OmpA was isolated and purified from the outer membrane of $E$. coli as described (1). DOPC and brominated phosphatidylcholines, 6,7-, 9,10-, and 11,12-DiBrPC were from Avanti Polar Lipids, Alabaster, AL. 4,5-DiBrPC as well as additional 9,10- and 11,12-DiBrPC, which were used for control experiments, was a generous gift from Dr. Peter Holloway (University of Virginia). All lipids were found to be pure by silica gel thin-layer chromatography using solvent systems $\mathrm{CHCl}_{3} / \mathrm{CH}_{3} \mathrm{OH} / \mathrm{NH}_{3}(\mathrm{aq})(65 / 30 / 3 \mathrm{v} / \mathrm{v} / \mathrm{v})$ and hexane/ ether $(1 / 1 \mathrm{v} / \mathrm{v})$.

Preparation of Small Unilamellar Vesicles. Solutions of DOPC $(10 \mathrm{mg} / \mathrm{mL})$ and $m, n$-DiBrPC $(5 \mathrm{mg} / \mathrm{mL})$ in chloroform were codissolved at $1 \mathrm{mg}$ total lipid, dried on the bottom of a glass test tube under a stream of nitrogen, and desiccated under high vacuum for at least $3 \mathrm{~h}$ to remove residual solvent. The lipids were dispersed in $0.5 \mathrm{~mL}$ of $10 \mathrm{mM}$ glycine buffer, $\mathrm{pH} 8.5$, containing $1 \mathrm{mM}$ EDTA and $150 \mathrm{mM} \mathrm{NaCl}$, to a lipid concentration of $2 \mathrm{mg} / \mathrm{mL}$. SUVs were prepared by sonication of the lipid dispersion for $50 \mathrm{~min}$ using the microtip of a Branson ultrasonifier at 50\% duty cycle in an ice/water bath. Titanium dust was removed by centrifugation at $735 \mathrm{~g}(3000 \mathrm{rpm})$ using a tabletop centrifuge (Eppendorff, Rexdale, Ontario). Five vesicle samples, consisting of pure DOPC and DOPC with $30 \mathrm{~mol} \%$ of each of the four different dibromo lipids, respectively, were sonicated sequentially using the same sonifier settings. Vesicles were then equilibrated overnight at $4{ }^{\circ} \mathrm{C}$ and used the day after preparation for fluorescence experiments. Samples, to be directly compared in fluorescence quenching profiles across the bilayer, were measured on the same day.

Folding and Membrane Insertion Detected by Fluorescence Spectroscopy. All fluorescence experiments were carried out at a molar lipid/protein ratio of 400 , with OmpA at a concentration of $1.4 \mu \mathrm{M}$ in a total volume of $1 \mathrm{~mL}$ of glycine buffer. Fluorescence measurements were performed on a SPEX Fluoromax spectrofluorometer (Spex Industries, Edison, NJ) and spectra recorded in the range of 310-370 $\mathrm{nm}$ with an increment of $0.5 \mathrm{~nm}$ and an integration time of $50 \mathrm{~ms}$. Excitation was at $290 \mathrm{~nm}$ using excitation and emission band-passes of 2.1 and $5 \mathrm{~nm}$, respectively. Three scans were averaged. Fluorescence units are in million counts per second (Mcps), and absolute intensities are directly comparable within this and the accompanying paper. Tryptophan $(2 \mu \mathrm{M})$ in glycine buffer produces $0.140 \mathrm{Mcps}$ at $355 \mathrm{~nm}$ with these experimental settings in our spectrofluorometer. The sample compartment was temperature-controlled, and the actual temperature of the sample was measured using a thermocouple and a digital thermometer (Omega, type HH21). Background spectra of the vesicles alone were taken first for later subtraction. Stock OmpA (10 $\mu \mathrm{L}$ ) in $8 \mathrm{M}$ urea solution were rapidly mixed into the cuvette at time 0 , and OmpA spectra were recorded immediately (i.e., the first at $30 \mathrm{~s}$ after mixing) and after specified time intervals. The sample was not illuminated between subsequent spectral recordings. Fluorescence data analysis was performed using the IGOR Pro 3.1 software (Wavemetrics, OR). Spectra were fitted to a log-normal distribution.

\section{RESULTS}

Fluorescence Quenching as a Function of Time and Temperature. We have performed fluorescence quenching experiments as function of time using four lipids brominated at the 4,5-, 6,7-, 9,10-, or 11,12-positions along the $s n-2$ acyl chain as depth-dependent quenchers in the lipid bilayer. The average positions of the bromine atoms have been determined by X-ray diffraction to be $12.8,11.0,8.3$, and $6.5 \AA$, respectively, from the center of the lipid bilayer (17). Fluorescence was monitored as a function of time after ureadenatured OmpA was mixed into a solution of preformed DOPC vesicles containing $30 \mathrm{~mol} \%$ of one of the brominated phosphatidylcholines (Figure 1). The same experiment was also carried out with pure DOPC vesicles lacking the quencher lipids (rightmost curve in Figure 1). In previous kinetic studies of OmpA refolding by fluorescence spectroscopy and SDS-PAGE, we identified two membrane-bound folding intermediates of OmpA (2). We found that different folding intermediates of OmpA could be stabilized at low temperatures for extended periods of time, with the first intermediate stabilized at $2{ }^{\circ} \mathrm{C}$. To probe the approach to this intermediate in terms of Trp location in the lipid bilayer, the experiments of Figure 1 were carried out at $2{ }^{\circ} \mathrm{C}$ in the absence and in the presence of each of the four brominated lipids used as fluorescence quenchers. Fluorescence ( $z$-axis) is plotted as a function of distance of the quenching bromine atoms from the bilayer center ( $x$-axis) and time ( $y$-axis). The fluorescence in the absence of quencher is plotted arbitrarily at the end of the $x$-axis. At $2{ }^{\circ} \mathrm{C}, 4,5-\mathrm{DiBrPC}$ quenched Trp fluorescence most effectively, followed by 6,7-, 9,10-, and 11,12-DiBrPC. This situation did not significantly change over a time period of $1 \mathrm{~h}$. Quenching by all membrane-bound quenchers was observed from the very beginning of the refolding experiment, indicating that OmpA adsorbs to the lipid bilayer surface within the first 1 or 2 min of mixing (see also ref 2). 


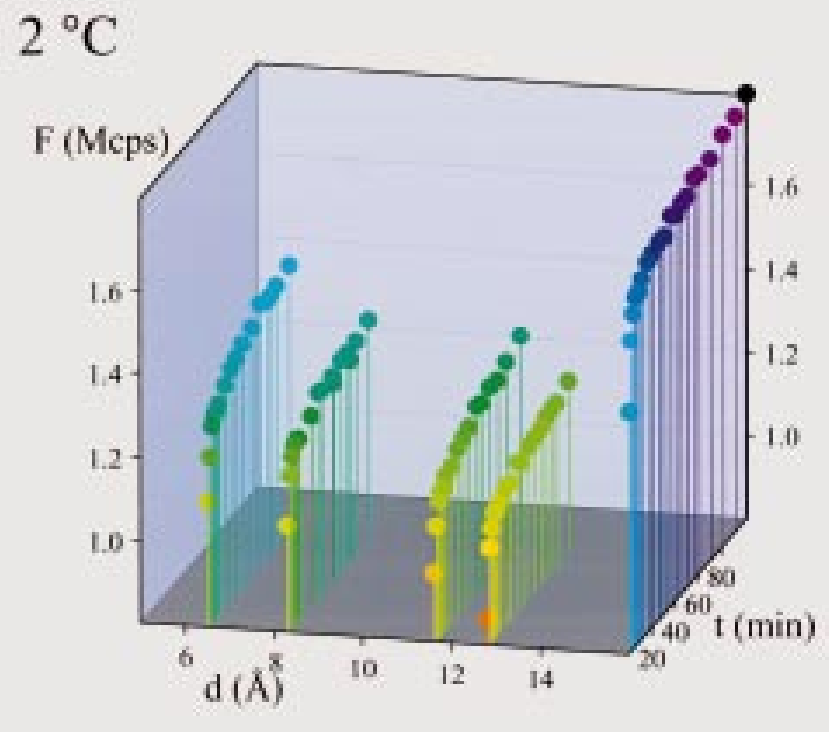

Figure 1: OmpA Trp fluorescence intensities at $330 \mathrm{~nm}$ as a function of time after being mixed with lipid vesicles and of distance of the quenching bromine atoms from the lipid bilayer center. At $2{ }^{\circ} \mathrm{C}$, OmpA adsorbs to the lipid bilayer surface and does not significantly penetrate with its Trp residues into the hydrophobic core of the lipid bilayer. Lipid vesicles composed of DOPC and $30 \mathrm{~mol} \%$ of either 11,12-, 9,10-, 6,7-, or 4,5-DiBrPC (curves from left to right) were used. The curve to the right was measured with pure DOPC vesicles. 4,5-DiBrPC is the strongest quencher of Trp fluorescence at all times in this experiment. The average distances of the bromines from the bilayer center (horizontal axis) are taken from the literature as described in the text. The quenching efficiency decreases with decreasing distance of the bromines from the bilayer center.

To probe the average Trp location of later intermediates on the folding pathway of OmpA, similar quenching experiments as a function of time were performed at 7, 20, 27.5, and $30{ }^{\circ} \mathrm{C}$ as shown in Figure 2. At 7 and $20{ }^{\circ} \mathrm{C}$ as well as at 15 and $26^{\circ} \mathrm{C}$ (data not shown), 6,7- and 9,10-DiBrPC were more effective quenchers than 4,5- and 11,12-DiBrPC, indicating a Trp location closer to the center of the lipid bilayer than observed at $2{ }^{\circ} \mathrm{C}$. Also, the relative differences between the quenching effects of the different brominated lipids did not change significantly between 7 and $26{ }^{\circ} \mathrm{C}$. However, at $27.5^{\circ} \mathrm{C}$, we found $11,12-\mathrm{DiBrPC}$ to be the most effective quencher, followed by 9,10-, 6,7-, and 4,5-DiBrPC. The same order of quenching efficiency occurred at $30{ }^{\circ} \mathrm{C}$ (and also at $40{ }^{\circ} \mathrm{C}$; see lower panels of Figure 4), but only during the first minutes. After several minutes at 30 or 40 ${ }^{\circ} \mathrm{C}$, the relative quenching efficiency of 9,10- and 6,7-DiBrPC became and remained more efficient whereas the quenching efficiency of 11,12-DiBrPC decreased.

Kinetics as a Function of Quencher Position and Temperature. The fluorescence kinetics, reflecting OmpA incorporation and refolding, are well represented by biexponential functions (Figure 3), i.e., a result that is consistent with those of our earlier study (2). At $2{ }^{\circ} \mathrm{C}$, characteristic times of $\tau_{1}=$ $2 \mathrm{~min}$ and $\tau_{2}=87 \mathrm{~min}$ were obtained from the fits. Even in the presence of membrane-bound quenchers the kinetics follow a biexponential time course at all temperatures. On the basis of this observation it is possible to estimate the initial and final extents of fluorescence quenching by membrane-incorporated quenchers at each temperature. These results are summarized in Table 1. The final extents of quenching of all membrane-bound quenchers were strongest at $20{ }^{\circ} \mathrm{C}$ and weaker at 2 and $40{ }^{\circ} \mathrm{C}$. The largest temperature effect was observed for 11,12-DiBrPC; fluorescence decreased by $25 \%$ from 2 to $20^{\circ} \mathrm{C}$ and increased by $23 \%$ from 20 to $40{ }^{\circ} \mathrm{C}$. The smallest differences between the different quenchers at infinite time were found at 27.5 ${ }^{\circ} \mathrm{C}$. However, the quenching efficiency at this temperature was also significantly weaker compared to that at $20^{\circ} \mathrm{C}$. Quite different quenching profiles were observed at an early time (2 min) after initiation of the refolding reaction (Table 1). In this case, the temperature where quenching was most efficient depends on the position of the bromine atoms. For example, the Trp fluorescence of OmpA was most strongly quenched by $4,5-\mathrm{DiBrPC}$ at $2{ }^{\circ} \mathrm{C}$, but for 11,12-DiBrPC the strongest quenching occurred at $40{ }^{\circ} \mathrm{C}$. Since the quenching efficiency depends on the number of fluorophores that are in close proximity to a given quencher position, it is obvious that the Trps of OmpA move from a surface location (at low temperatures and early times) to a deeply membraneembedded position (at intermediate temperatures and long times or at high temperatures and short times) and finally back to a surface location (at high temperatures and long times).

Determination of the Average Tryptophan Location by Distribution Analysis. To estimate the average position of the Trp residues of OmpA from the bilayer center, the fluorescence intensities measured in the presence of quenchers were first normalized relative to the fluorescence intensities in the absence of quenchers. Two methods have been developed previously to determine fluorophore positions in lipid bilayers from such data, namely, the parallax method (20) and distribution analysis $(21,22)$. The parallax method relies on distance geometry and allows one to determine the position of the fluorophore from any pair of membrane-bound quenchers. This method has also been extended to include quenchers that are covalently linked to the lipid headgroup (23). In distribution analysis, at least three membrane-bound quenchers are needed to calculate the average position of fluorophores. The accuracy of the method is improved as more quenchers at different depths in the membrane are used. In addition to the average position, the distribution analysis method also yields a measure of the width of the distribution of the fluorophore in the lipid bilayer. Distribution analysis assumes a Gaussian distribution of distances between the quencher and the fluorophore and an exponential decay of the quenching efficiency as a function of this distance (22):

$$
\frac{F\left(d_{\mathrm{Q}}\right)}{F_{0}}=\exp \left\{-\frac{S}{\sigma \sqrt{2 \pi}} \exp \left\{-\frac{1}{2}\left(\frac{d_{\mathrm{Q}}-d_{\mathrm{Trp}}}{\sigma}\right)^{2}\right\}\right\}
$$

$F_{0}$ and $F\left(d_{\mathrm{Q}}\right)$ in eq 1 are the fluorescence intensities in the absence and in the presence of a particular $m, n$-DiBrPC quencher. $d_{\mathrm{Q}}$ is the distance of the quencher from the lipid bilayer center, and $d_{\operatorname{Trp}}$ is the distance of the fluorophore (Trp) from the center. The dispersion, $\sigma$, is a function of the sizes of the fluorophore and quencher and thermal fluctuations between the two. $S$ is a function of the quenching efficiency and quencher concentration in the membrane. Equation 1 has been derived for samples that are in thermal equilibrium. Here, we investigate average Trp positions as a function of time during refolding of OmpA. Obviously, in a kinetic process such as the one considered here, a nonequilibrium distribution of Trp distances from the bilayer 

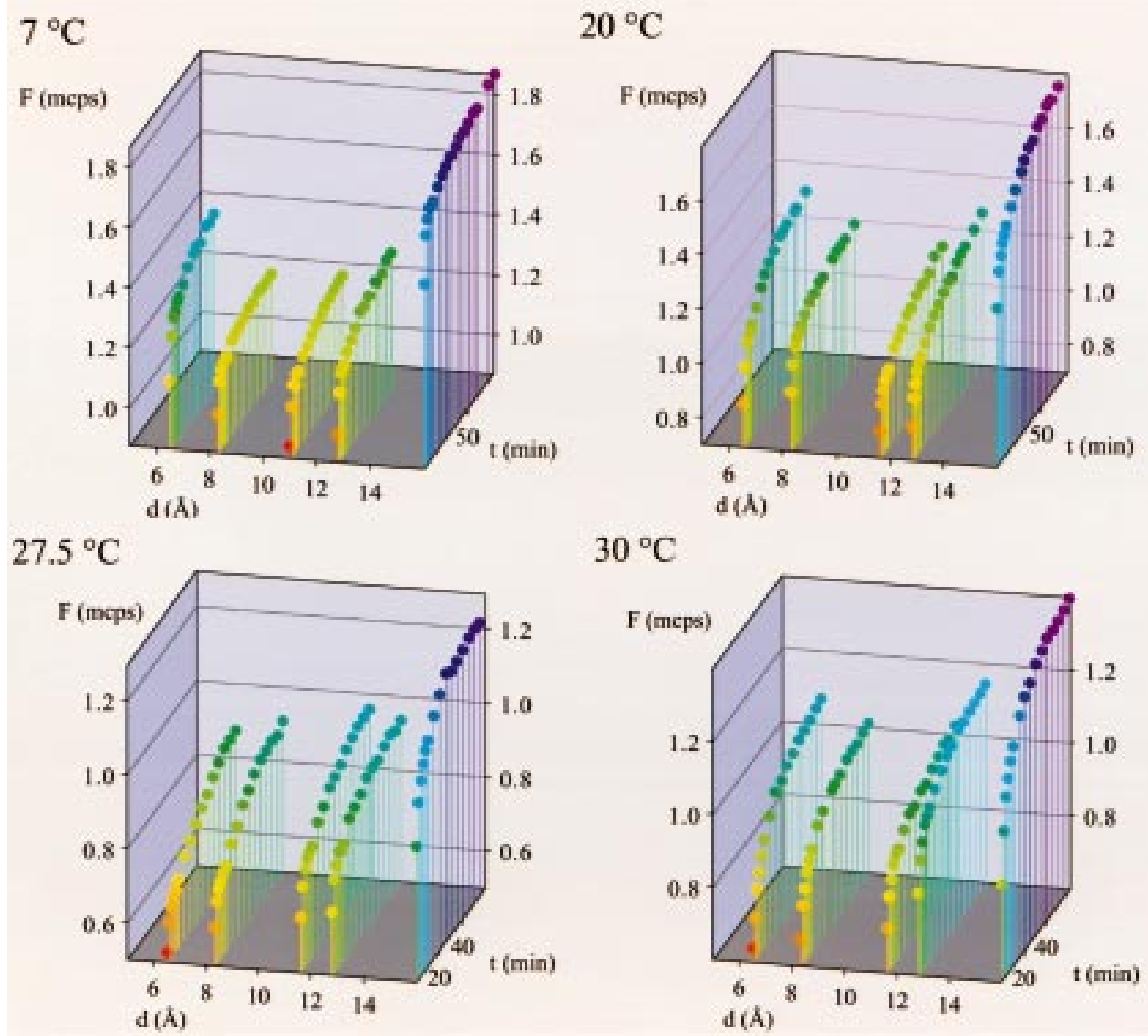

FIgURE 2: OmpA Trp fluorescence intensities at $330 \mathrm{~nm}$ monitored at 7, 20, 27.5, and $30^{\circ} \mathrm{C}$ as a function of time and bromine distance from the lipid bilayer center. All conditions, except temperature, are as in Figure 1. Trp fluorescence is progressively more quenched by the deeper quenchers, indicating decreasing distances of the Trps from the bilayer center as the temperature is raised to $27.5^{\circ} \mathrm{C}$. At $30{ }^{\circ} \mathrm{C}$, the 11,12-DiBrPC is initially the most efficient quencher, but as time progresses 9,10-DiBrPC and 6,7-DiBrPC become more efficient quenchers.

center will exist. At a given time, some proteins may have progressed more than others along the reaction coordinate. However, their distributions in several positions along the reaction coordinate may still be Gaussian in this scenario. In fact, we found that some quenching profiles are quite symmetric and Gaussian around the mean. Therefore and despite the nonequilibrium conditions of our experiments, we have assumed Gaussian distribution in the analysis of our data. ${ }^{2}$

Figure 4 shows the results of fitting fluorescence quenching profiles, $F\left(d_{\mathrm{Q}}\right) / F_{0}$, at $2{ }^{\circ} \mathrm{C}$ and at $40{ }^{\circ} \mathrm{C}$ at various times during the refolding reaction. At $40^{\circ} \mathrm{C}$, the profiles are well described by Gaussian distributions. The average Trp location is given by the minimum of the fit function (solid curves in Figure 4). These minima are clearly changing over time. The most dramatic change in quenching efficiency was observed between 2 and $30 \mathrm{~min}$ after refolding for 11,12-DiBrPC $\left[F\left(d_{\mathrm{Q}}\right) / F_{0}\right.$ increased by 0.2$]$ while relative changes were small

${ }^{2}$ We would like to emphasize that, by treating the data as Gaussian distributions, we do not imply that the distributions are in fact Gaussian at all positions along the reaction coordinate. Most likely, they are not Gaussian in many situations, but the Gaussian distributions seem to contribute mostly to observable profiles. within the first $2 \mathrm{~min}$ and after $30 \mathrm{~min}$. Similar observations were made for the quenching efficiency of 9,10-DiBrPC, for which $F\left(d_{\mathrm{Q}}\right) / F_{0}$ increased by 0.13 between 2 and $30 \mathrm{~min}$, whereas small changes were observed initially and after 60 $\mathrm{min}$. The relative fluorescence quenching of 6,7-DiBrPC and 4,5-DiBrPC remained unchanged or decreased with time. Calculations of the Trp distances from the bilayer center, $d_{\text {Trp }}$, from these data show that the Trps moved from an average distance of $7 \AA$ to a distance of $9.7 \AA$ from the center within $1 \mathrm{~h}$ at $40{ }^{\circ} \mathrm{C}$. At $2{ }^{\circ} \mathrm{C}$, average Trp distances could only be estimated from extrapolation. The data show relatively small changes in quenching efficiency compared to those measured at $40{ }^{\circ} \mathrm{C}$ over the same time period. Many experiments performed at other temperatures were well fitted using the Gaussian distribution. To provide a sense for the goodness of these fits, we found that the errors for the average Trp location are $7.1 \pm 0.5 \AA$ after $20 \mathrm{~s}$ and $9.7 \pm$ $0.3 \AA$ after $60 \mathrm{~min}$ at $40{ }^{\circ} \mathrm{C}$ (Figure 4). With the exception of experiments performed at 27.5 or, during initial times, at $28{ }^{\circ} \mathrm{C}$, the average $\operatorname{Trp}$ distances could be obtained from interpolation rather than extrapolation (see also Figure 2). To check for possible effects due to transbilayer quenching, we also evaluated all data with a modified distribution 


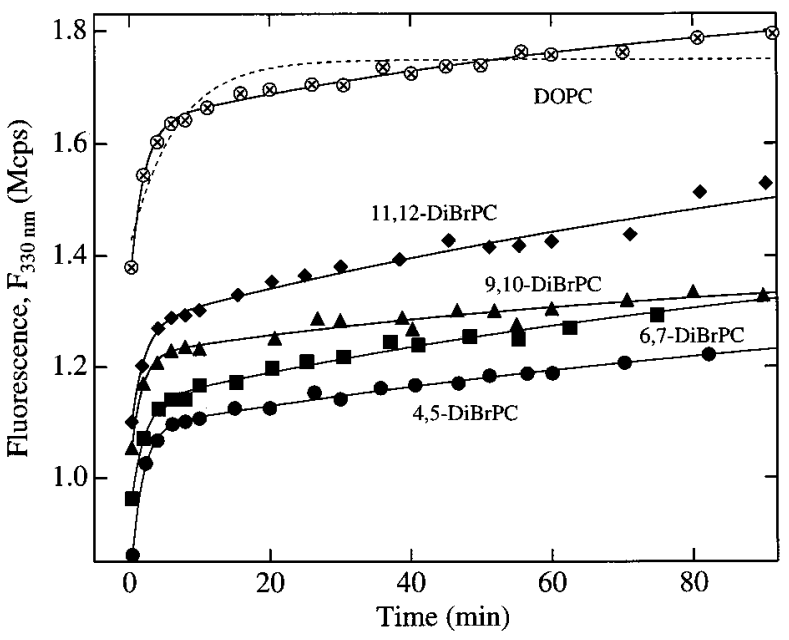

FIGURE 3: The kinetics of OmpA insertion into DOPC bilayers as monitored by Trp fluorescence follow double exponential time courses in the presence or absence of $30 \mathrm{~mol} \% m, n$-DiBrPC as indicated. All measurements were at $2{ }^{\circ} \mathrm{C}$. The characteristic times and relative amplitudes were $A_{1}=-0.3, \tau_{1}=1.9 \mathrm{~min}^{-1}, A_{2}=$ $-0.25, \tau_{2}=84 \mathrm{~min}^{-1}$ (DOPC), $A_{1}=-0.3, \tau_{1}=1.7 \mathrm{~min}^{-1}, A_{2}=$ $-0.28, \tau_{2}=132 \mathrm{~min}^{-1}$ (4,5-DiBrPC), $A_{1}=-0.21, \tau_{1}=1.9 \mathrm{~min}^{-1}$, $A_{2}=-0.34, \tau_{2}=111 \mathrm{~min}^{-1}$ (6,7-DiBrPC), $A_{1}=-0.21, \tau_{1}=1.5$ $\min ^{-1}, A_{2}=-0.16, \tau_{2}=79 \mathrm{~min}^{-1}(9,10-\mathrm{DiBrPC})$, and $A_{1}=-0.21$, $\tau_{1}=1.9 \mathrm{~min}^{-1}, A_{2}=-0.43, \tau_{2}=125 \mathrm{~min}^{-1}(11,12-\mathrm{DiBrPC})$. The dashed line represents a single exponential fit for comparison.

Table 1: Initial and Final Extent of Fluorescence Quenching at Various Temperatures ${ }^{a}$

\begin{tabular}{ccccc}
\hline & \multicolumn{4}{c}{$m, n$-DiBrPC } \\
\cline { 2 - 5 }$T\left({ }^{\circ} \mathrm{C}\right)$ & 4,5 & 6,7 & 9,10 & 11,12 \\
\hline \multicolumn{5}{c}{ Relative Fluorescence, $F_{\mathrm{Q}} / F_{0}$, at $t=2$ min } \\
2 & 0.65 & 0.70 & 0.76 & 0.80 \\
7 & 0.67 & 0.63 & 0.67 & 0.76 \\
20 & 0.65 & 0.63 & 0.72 & 0.70 \\
27.5 & 0.77 & 0.74 & 0.68 & 0.62 \\
30 & 0.90 & 0.80 & 0.75 & 0.72 \\
40 & 0.81 & 0.72 & 0.54 & 0.58 \\
\multicolumn{5}{c}{} \\
2 & Relative Fluorescence, $F_{\mathrm{Q}} / F_{0}$, extrapolated to $t=\infty$ \\
7 & 0.74 & 0.80 & 0.75 & 0.94 \\
20 & 0.70 & 0.67 & 0.65 & 0.77 \\
27.5 & 0.69 & 0.62 & 0.64 & 0.70 \\
30 & 0.75 & 0.78 & 0.73 & 0.75 \\
40 & 0.82 & 0.76 & 0.73 & 0.79 \\
& 0.80 & 0.75 & 0.71 & 0.86 \\
\hline
\end{tabular}

${ }^{a}$ The standard errors of the data at 2 min are between $1 \%$ and $3 \%$, and those of the data at infinite time are between $15 \%$ and $25 \%$. Errors intermediate between these two extremes are found at intermediate times.

analysis which takes this effect into account (24), but we did not observe any significant differences in the fitting parameters between the standard and this extended method.

Tryptophan Distances as a Function of Time. Figure 5 shows the average Trp distances calculated by distribution analysis as a function of time at temperatures between 2 and $26{ }^{\circ} \mathrm{C}$. At $2{ }^{\circ} \mathrm{C}$, the extrapolated average Trp distance followed a single exponential time course, with the Trps approaching a distance of $14-16 \AA$ from the bilayer center 10 min after initiation of refolding. Between 7 and $26^{\circ} \mathrm{C}$ and after $1 \mathrm{~h}$, an average Trp distance of 10-11 $\AA$ from the center was calculated. The expanded distance scales in the insets show that the Trp residues approached this average distance from larger distances. The time courses of the Trp movements relative to the bilayer center were best fit to

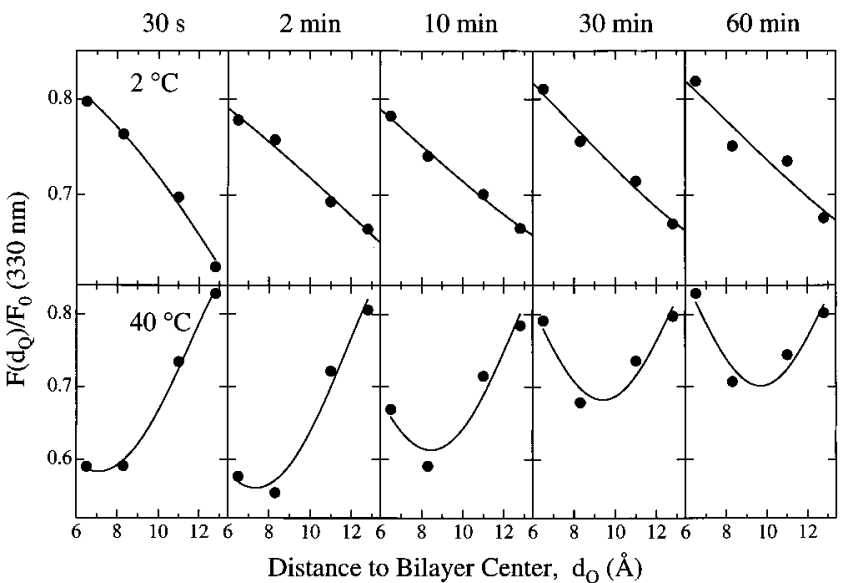

FIGURE 4: Fluorescence quenching profiles across half-bilayers at $2{ }^{\circ} \mathrm{C}$ (upper panels) and at $40{ }^{\circ} \mathrm{C}$ (lower panels) after $30 \mathrm{~s}$ and 2 , 10,30 , and $60 \mathrm{~min}$ of OmpA incorporation. The profiles are generated by plotting the fluorescence ratios $F\left(d_{\mathrm{Q}}\right) / F_{0}$ as a function of the quencher distance from the bilayer center. The solid lines represent best fits of the data to eq 1 . The minima in the lower panels indicate the mean position of the Trps of OmpA in each case.

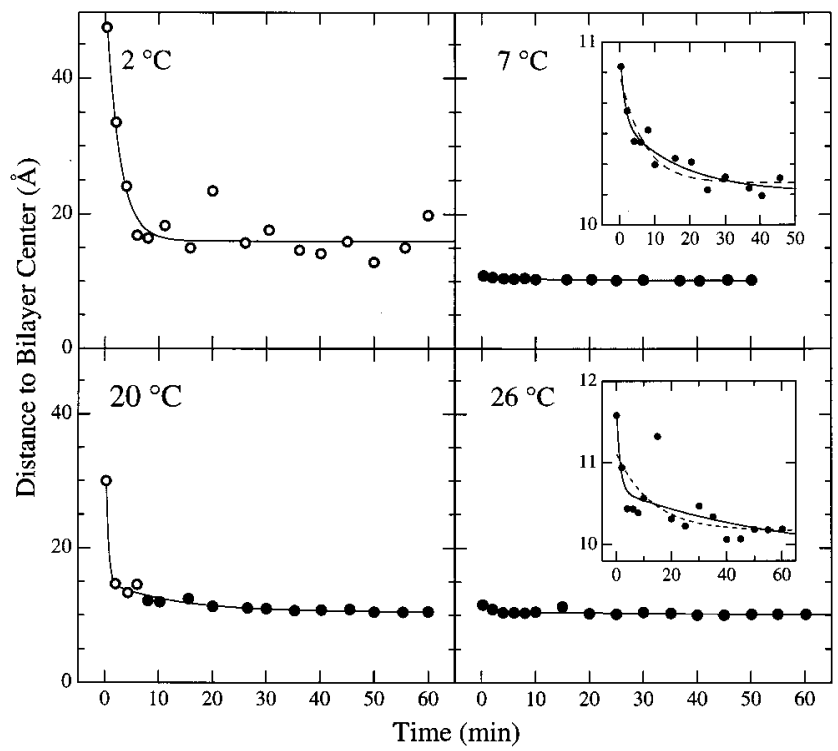

FIGURE 5: Time courses of Trp movement toward the bilayer center at $2,7,20$, and $26{ }^{\circ} \mathrm{C}$. Distances were obtained from curve fits of the fluorescence quenching profiles as shown in Figure 4 . The insets at 7 and $26^{\circ} \mathrm{C}$ show the data on expanded distance scales. Data points represented by closed circles were obtained from observed quenching minima; open circles denote extrapolated distances from the observed quenching profiles. The kinetics of Trp translocation into the bilayer were monoexponential at $2{ }^{\circ} \mathrm{C}$ and biexponential at 7,20 , and $26^{\circ} \mathrm{C}$.

biexponential functions at these temperatures. At $27.5^{\circ} \mathrm{C}$ and shortly after initiation of refolding at $28^{\circ} \mathrm{C}$, the deepest quenchers were most efficient and the quenching profiles located the Trps to the center of the bilayer (Figure 6). This situation was stable for more than $1 \mathrm{~h}$ at $27.5^{\circ} \mathrm{C}$. At $28{ }^{\circ} \mathrm{C}$, the Trps were centrally located for the first $6 \mathrm{~min}$, after which time they moved out to an average distance of $9-10 \AA$ from the bilayer center. At still higher temperatures (30 and 40 ${ }^{\circ} \mathrm{C}$ ), the Trps moved from 7 to 8 to $10 \AA$, and the initial location close to the center was no longer observed at our current time resolution of about $1 \mathrm{~min}$. Again, the time dependences of Trp distance are best fit using biexponential functions at all temperatures, except at $40{ }^{\circ} \mathrm{C}$, where a single 


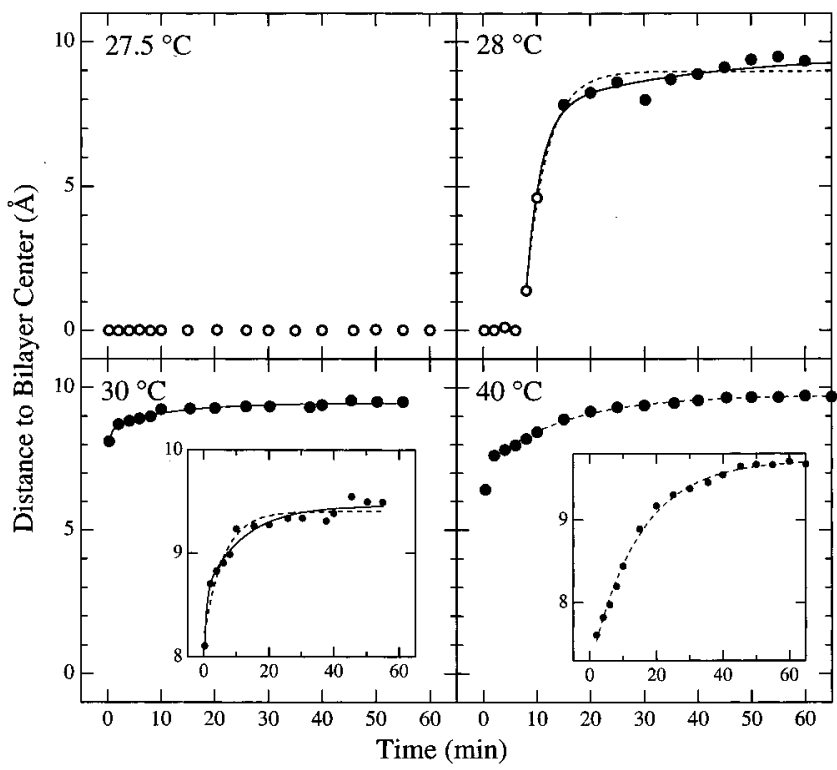

FIGURE 6: Time courses of Trp movement from the center toward the surface of the bilayer at $27.5,28,30$, and $40{ }^{\circ} \mathrm{C}$. The data were obtained and analyzed as those of Figure 5. The kinetics of Trp translocation from the bilayer center toward the surface were fit to biexponential (solid lines) or monoexponential (dashed lines) functions.

Table 2: Rate Constants of Trp Translocation across DOPC Lipid Bilayers

\begin{tabular}{|c|c|c|c|c|c|c|}
\hline \multirow[b]{2}{*}{$T\left({ }^{\circ} \mathrm{C}\right)$} & \multicolumn{3}{|c|}{ cis monolayer $^{a}$} & \multicolumn{3}{|c|}{ trans monolayer } \\
\hline & $A_{t, 1}{ }^{c}$ & $\begin{array}{l}\vec{k}_{t, 1}{ }^{d}\left(\min ^{-1}\right) \\
\AA^{b}\end{array}$ & $\begin{array}{c}\rightarrow 10 \AA \\
k_{t, 2}\left(\min ^{-1}\right)\end{array}$ & $A_{t, 3}$ & $\begin{array}{c}\rightarrow 8 \AA \\
k_{t, 3}\left(\min ^{-1}\right)\end{array}$ & $\begin{array}{c}\rightarrow 10 \AA \\
k_{t, 4}\left(\min ^{-1}\right)\end{array}$ \\
\hline 2 & 1.0 & $0.40 \pm 0.07$ & no ${ }^{e}$ & no & no & no \\
\hline 7 & 0.5 & $0.8 \pm 0.7$ & $0.06 \pm 0.04$ & no & no & no \\
\hline 20 & 0.9 & $2.3 \pm 1.6$ & $0.08 \pm 0.03$ & no & no & no \\
\hline 26 & 0.6 & $0.8 \pm 0.8$ & $0.02 \pm 0.04$ & no & no & no \\
\hline 27.5 & $\mathrm{~s}^{e}$ & $\mathrm{~s}$ & $\mathrm{~s}$ & $\mathrm{~s}$ & $\mathrm{~s}$ & $\mathrm{~s}$ \\
\hline 28 & no & no & no & 0.98 & $0.37 \pm 0.09$ & $0.04 \pm 0.04$ \\
\hline 30 & no & no & no & 0.5 & $1.5 \pm 1.9$ & $0.08 \pm 0.03$ \\
\hline 40 & no & no & no & 1 & no & $0.09 \pm 0.01$ \\
\hline
\end{tabular}

${ }^{a}$ For assignment, see Discussion. ${ }^{b}$ Approximate Trp distances from the bilayer center in the intermediates formed at each step. ${ }^{c}$ Relative amplitudes for faster process. ${ }^{d}$ Translocation rate constants. ${ }^{e}$ no, not observed; s, stationary.

exponential function was sufficient to describe all but the first data points (see inset).

Table 2 summarizes the rate constants obtained from fits of the Trp distances $v s$ time as shown in Figures 5 and 6. Four different steps (with different time constants and Trp positions) in the refolding reaction are easily distinguished: (1) The protein adsorbs quickly to the membrane surface. The characteristic time $\tau_{\text {Trp }, 1}=2.5 \min \left(k \approx 0.4 \mathrm{~min}^{-1}\right)$ of this process coincides with the characteristic time of the first step at $2{ }^{\circ} \mathrm{C}$ that was obtained by directly analyzing the fluorescence kinetics $\left(\tau_{1}=2 \mathrm{~min}\right)$. (2) At $7{ }^{\circ} \mathrm{C}$, a double exponential time course is observed for the Trp movements into the lipid bilayer. While the rate of the first process increases about 2 -fold (compared to $2{ }^{\circ} \mathrm{C}$ ), the onset of a second process with a time constant $\tau_{\operatorname{Trp}, 2}=16.7 \mathrm{~min}$ is observed at this temperature. (3) No change in Trp location is observed at $27.5^{\circ} \mathrm{C}$. The average position of the Trps is close to the bilayer center under these conditions. (4) At the higher temperatures $\left(28-40{ }^{\circ} \mathrm{C}\right)$, the Trps move from a location near the bilayer center closer to the bilayer surface.
Trp movement toward the bilayer center, as observed below $27.5^{\circ} \mathrm{C}$, is no longer observed at these higher temperatures. The first two steps have become too fast to be observable, and we therefore resolve only steps 3 and 4 . The third step is best resolved at $28{ }^{\circ} \mathrm{C}$ and has a characteristic time $\tau_{\operatorname{Trp}, 3}$ $=2.7 \mathrm{~min}$. The fourth step is slower with $\tau_{\operatorname{Trp}, 4}=25 \mathrm{~min}$. At 30 and $40{ }^{\circ} \mathrm{C}, \tau_{\operatorname{Trp}, 3}$ becomes smaller and only step 4 can be resolved. Step 4 remains slow at $\tau_{\text {Trp }, 4}=11-16 \mathrm{~min}$.

Distribution Parameters $\sigma$ and $S$ as a Function of Time and Temperature. Figure 7 shows the fit parameters $\sigma$ and $S$ obtained from eq 1 as a function of time and temperature. $\sigma$ is the dispersion (width at half-height) of the Gaussian distribution (22), and $S$ is a quenching amplitude, which depends on the concentration of quencher close to the fluorophore and on the quenching mechanism. The large dispersion found initially at $2{ }^{\circ} \mathrm{C}$ is decreasing with time, indicating an increasingly narrower distance distribution of the Trp residues in OmpA. At $7{ }^{\circ} \mathrm{C}$, the values of $\sigma$ are much smaller, i.e., $4.5 \AA$ in the beginning and $3.5 \AA 1 \mathrm{~h}$ after mixing. These narrower distributions are comparable to those found for Trp residues of native integral membrane proteins (22). Similar reductions of the dispersions were obtained at 30 and $40{ }^{\circ} \mathrm{C}$. However, at $27.5^{\circ} \mathrm{C}$ and initially at $28^{\circ} \mathrm{C}, \sigma$ increases with time, indicating increasingly larger Trp distributions. At $2{ }^{\circ} \mathrm{C}$ the amplitude parameter $S$ varied over a wide range and stabilized only after $1 \mathrm{~h}$ at values of 5-10 $\AA$ A. At temperatures 7,30 , and $40{ }^{\circ} \mathrm{C}, S$ decreased more monotonically with time, but at $27.5^{\circ} \mathrm{C}, S$ remained constant at a higher value.

Acrylamide Quenching Experiments. To probe Trp accessibility from the aqueous phase, we also performed quenching experiments with acrylamide as a soluble quencher. Figure 8 shows Stern-Volmer plots of OmpA in glycine buffer and after incubation with DOPC vesicles at 2 and at $40{ }^{\circ} \mathrm{C}$. At $2{ }^{\circ} \mathrm{C}$, after $10 \mathrm{~min}$, quenching of OmpA fluorescence in the presence of DOPC was not significantly different from quenching in the absence of vesicles. In contrast, at $40{ }^{\circ} \mathrm{C}$, after $1 \mathrm{~h}$ of incubation, OmpA was mostly protected from acrylamide quenching. The data were fitted to the linear Stern-Volmer function. Stern-Volmer constants of $5.3 \mathrm{M}^{-1}$ (glycine buffer, $2{ }^{\circ} \mathrm{C}$ ), $5.4 \mathrm{M}^{-1}$ (DOPC, $2{ }^{\circ} \mathrm{C}$ ), $11.7 \mathrm{M}^{-1}$ (glycine buffer, $40{ }^{\circ} \mathrm{C}$ ), and $1.8 \mathrm{M}^{-1}$ (DOPC, $40{ }^{\circ} \mathrm{C}$ ) were derived from the slopes of these fits.

\section{DISCUSSION}

In this work, we have presented a new technique, timeresolved distance determination by fluorescence quenching (TDFQ), which enabled us to follow the translocation of tryptophans in polypeptide chains across lipid bilayers in real time and at relatively high spatial resolution. Specifically, we have shown that we can monitor the trans bilayer movement of tryptophans across lipid bilayers of outer membrane protein A when unfolded protein is reacted with small unilamellar vesicles under refolding conditions. The technique uses covalently linked quenchers (bromine atoms) at different depths in the lipid bilayer. With the probes that were available to us, we could monitor with high precision Trp movements in both $5-15 \AA$ zones from the bilayer center, but movements at other depths in the bilayer could also be estimated from extrapolations of the data. We are not aware of other techniques by which the same kind of 


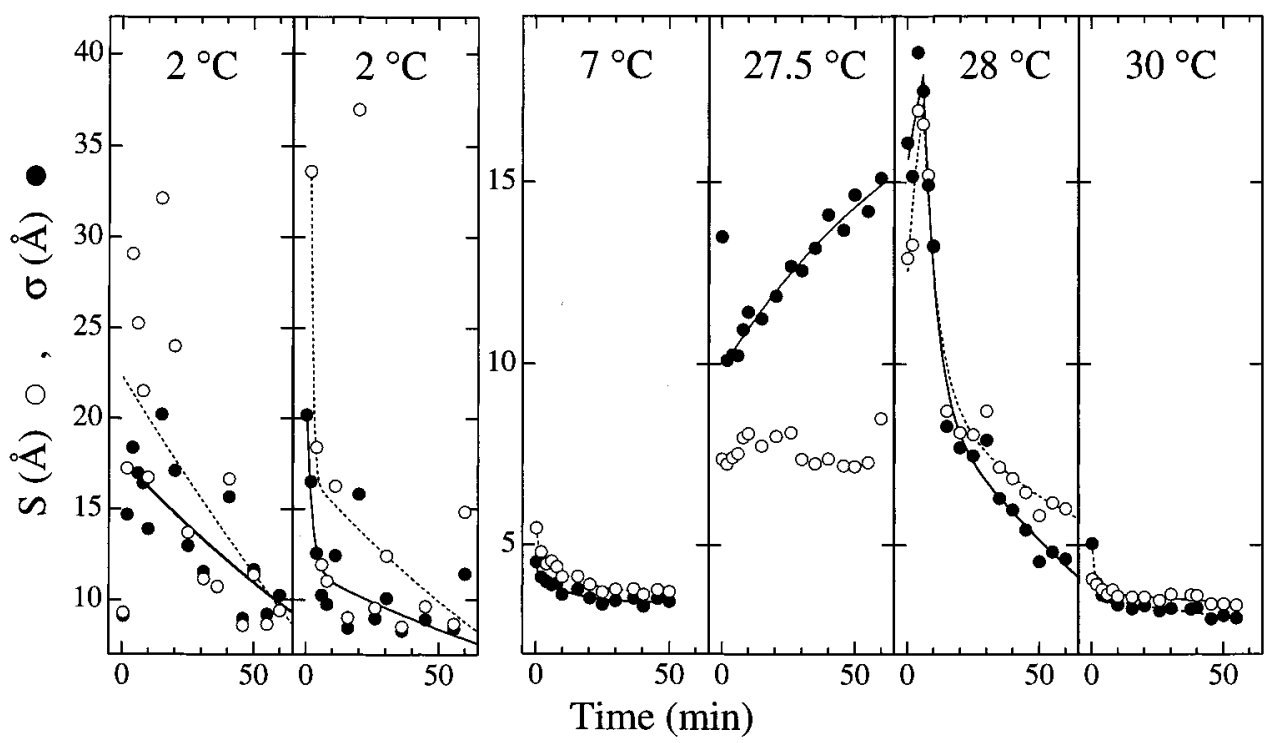

FIGURE 7: Time courses of the fit parameters $\sigma$, representing the widths of Gaussian distributions of the Trps (filled circles), and $S$, which is a measure of the quenching efficiency (open circles), obtained from fluorescence quenching profiles such as those shown in Figure 4 during OmpA refolding in lipid bilayers at $2,7,27.5,28$, and $30^{\circ} \mathrm{C}$. The Trp distributions become narrower as folding proceeds at all temperatures, except at $27.5^{\circ} \mathrm{C}$, where the Trp distribution becomes very broad with time.

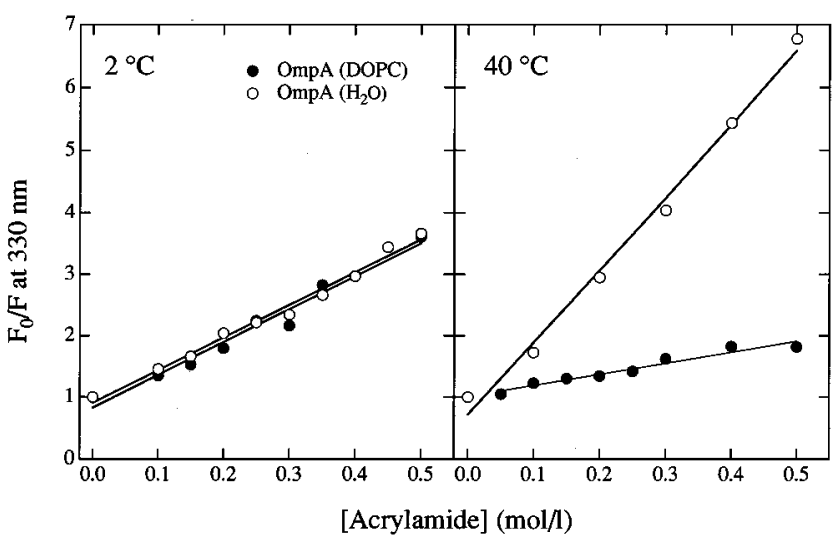

FIGURE 8: Stern-Volmer plots of OmpA Trp fluorescence quenching by acrylamide at $2{ }^{\circ} \mathrm{C}$ (left panel) and at $40{ }^{\circ} \mathrm{C}$ (right panel) in buffer (open circles) and after binding to DOPC bilayers (filled circles). $F$ and $F_{0}$ are the fluorescence intensities in the presence and in the absence of acrylamide, respectively. $F$ has been corrected for the inner filter effect for absorption, $F_{\text {corr }}=F_{\text {meas }} \times$ $10^{1 / 2(0.55 \text { [acrylamide] })}$. The inner filter effect at the emission wavelength was negligible. The derived Stern-Volmer constants are given in the text.

information could have been obtained with similar or better spatial resolution in the transbilayer dimension.

The experiments of this work allowed us to dissect several steps in the refolding process of OmpA. A previous study showed that folding of OmpA into lipid bilayers is strongly temperature-dependent and involves several membranebound intermediates (2). In fact, folding in DOPC does not go to completion at temperatures below approximately 27 ${ }^{\circ} \mathrm{C}$. Here, we show that the refolding process in DOPC can be subdivided into two major regimes, i.e., one regime during which the Trps of OmpA move toward the center of the bilayer and another regime during which they move out toward the membrane surface. Our method does not provide direct information on the sidedness of the movement of the polypeptide chain; i.e., we cannot unambiguously decide whether the Trps move in the second regime from the bilayer center toward the surface of the trans monolayer or back toward the cis monolayer. This question could potentially be resolved with quenchers that are asymmetrically distributed across the lipid bilayer. Although we tried many different approaches, we were unsuccessful in maintaining asymmetry of lipid-bound or soluble quenchers or resonance energy transfer probes for the entire duration of a typical folding experiment. ${ }^{3}$ Similar observations were made recently with a pore-forming peptide (25). Despite this lack of direct evidence, it is most reasonable to conclude that the inward movement seen in the early and lower temperature regime represents the passage of most Trps through the cis monolayer and the outward movement seen later and at the higher temperatures represents the passage of the same Trps across the trans monolayer, which will be required to achieve the native transmembrane topology of this protein. This interpretation is also consistent with the results obtained with the five single Trp mutants of OmpA (see accompanying paper). The inward moving regime dominates at temperatures $<27.5$ ${ }^{\circ} \mathrm{C}$, and the outward moving regime takes over at temperatures $>27.5{ }^{\circ} \mathrm{C}$. In agreement with our previous results, completely inserted and fully refolded OmpA is only found after about 30-60 min incubations at the higher temperatures.

In a previous study, we monitored the refolding kinetics of OmpA in the absence of positional quenchers (2). These kinetics followed double exponential time courses, which indicated folding intermediates with different fluorescence emissions. The Trp movements into and across the lipid bilayer as determined in this work by TDFQ were also best characterized by double exponential time courses. However, it should be noted that the two types of experiments monitor two fundamentally different processes with rates that are not comparable. In the binding and insertion experiments, we measure true chemical rate constants which characterize the conversion between two or more chemically distinct states.

\footnotetext{
${ }^{3}$ Pure lipid bilayers with an asymmetric lipid distribution can be maintained for extended periods of time. The problem is the insertion of OmpA which collapses transbilayer gradients and scrambles transbilayer lipid asymmetry.
} 


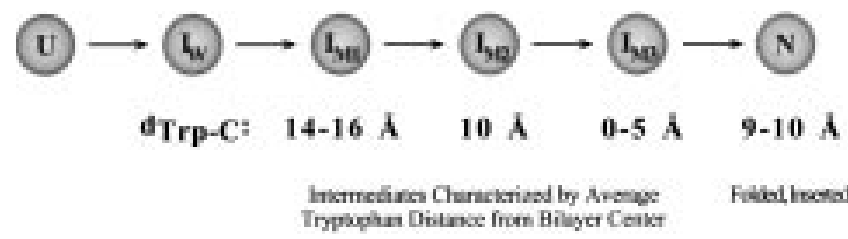

FIGURE 9: Sequence of observed intermediates on the membrane insertion and folding pathway of OmpA. Unfolded OmpA (U) hydrophobically collapses in a kinetically unresolved step to a water-soluble intermediate $\left(\mathrm{I}_{\mathrm{W}}\right)$ which adsorbs to the lipid bilayer surface (intermediate $\mathrm{I}_{\mathrm{M} 1}$ ) where the Trp residues are located on average 14-16 $\AA$ from the bilayer center. This intermediate is stable at $2{ }^{\circ} \mathrm{C}$ for at least $60 \mathrm{~min}$. Folding proceeds to a second membranebound intermediate $\left(\mathrm{I}_{\mathrm{M} 2}\right)$ with its Trps at an average distance 10$11 \AA$ from the bilayer center when the temperature is kept between $\sim 7$ and $\sim 25{ }^{\circ} \mathrm{C}$. A third intermediate $\left(\mathrm{I}_{\mathrm{M} 3}\right)$ with an average Trp distance $<5 \AA$ from the bilayer center appears at 27 to $28^{\circ} \mathrm{C}$. In the last step the Trps are completely translocated across the lipid bilayer and a structure is formed that by all available criteria is indistinguishable from the native, fully membrane-inserted state $(\mathrm{N})$ of OmpA.

In contrast, the translocation rates obtained from TDFQ reflect velocities (distance/time) of the Trp trajectories projected onto the normal of the lipid bilayer. These velocities are retarded by friction and other interactions in the lipid bilayer until they reach 0 , when the final equilibrium state is approached. Therefore, the rate constants obtained from TDFQ should be viewed as kinematic rather than pure chemical reaction rate constants.

Depending on the temperature at which refolding was performed, the TDFQ measurements allowed us to differentiate between four relatively stable distances of the average Trp location from the bilayer center. Therefore, instead of two membrane-bound folding intermediates which were deduced from the previous temperature-dependent fluorescence kinetics, we were now able not only to identify but also to characterize by TDFQ three such intermediates and the final native state of OmpA (Table 2 and Figure 9). The hydrophobic collapse of OmpA to an aqueous intermediate, $\mathrm{I}_{\mathrm{W}}$, after rapid dilution of the urea was still not resolved, because it takes place in the aqueous space which is too distant from the membrane-bound quenchers. A second reason for not resolving this step is that, as in our previous study (2), the hydrophobic collapse is fast and all our experiments were performed by hand mixing of the reactants.

The first membrane-bound intermediate, $\mathrm{I}_{\mathrm{M} 1}$, is found about $1 \mathrm{~min}$ after initiation of OmpA refolding in the presence of DOPC vesicles. The Trps are located on average 14-16 $\AA$ from the bilayer center in this intermediate. This intermediate appears to be stabilized at $2{ }^{\circ} \mathrm{C}$. At temperatures between 7 and $26{ }^{\circ} \mathrm{C}$, OmpA progresses to a second membrane-bound intermediate, $\mathrm{I}_{\mathrm{M} 2}$, which is characterized by a deeper average Trp location, i.e., approximately $10 \AA$ from the bilayer center. This state is acquired in $12-50 \mathrm{~min}$, depending on temperature. In previous temperature jump experiments we showed that $\mathrm{I}_{\mathrm{M} 1}$ and (as we now know) $\mathrm{I}_{\mathrm{M} 2}$, are true folding intermediates rather than side products that occurred only at low temperatures (2). Temperature jump experiments with brominated lipids confirmed this earlier conclusion (data not shown).

A third membrane-bound intermediate, $\mathrm{I}_{\mathrm{M} 3}$, with an average Trp distance $<5 \AA$ from the bilayer center could be isolated at $27.5^{\circ} \mathrm{C}$. Extrapolation of the quenching profile predicts the average Trp location of this intermediate very close to the center of the bilayer. This intermediate is also observed for a few minutes at $28{ }^{\circ} \mathrm{C}$, but then the Trps move from this deep location to about $10 \AA$, presumably on the trans side from the bilayer center. This process is biexponential at $30^{\circ} \mathrm{C}$ but monoexponential, requiring about $10-$ $20 \mathrm{~min}$, at $40^{\circ} \mathrm{C}$. As judged by protection from protease digestion, this refolded form is indistinguishable from the native state, $\mathrm{N}$, of OmpA (2). The average distance of the Trp backbone position from the bilayer center in the native structure predicted by Vogel and Jähnig (5) is about $15 \AA$. Taking into account the size and flexibility of Trp side chains, their prediction is not inconsistent with our experimental average distance.

Previously, we observed kinetic rate constants for the insertion of OmpA in DOPC bilayers without spatial resolution (2). We reported a temperature-"independent" step and a temperature-dependent step, which were characterized by rate constants of $0.16 \mathrm{~min}^{-1}$ and $0.006-0.04 \mathrm{~min}^{-1}(2-40$ $\left.{ }^{\circ} \mathrm{C}\right)$, respectively. As we noted above, these rate constants should not be directly compared to the translocation rate constants reported in Table 2 because these latter constants refer to translocation rates of spatially resolved steps, whereas the previously reported rates each refer to several processes integrated over all distances that result in the same fluorescence change. The Trps in intermediate $\mathrm{I}_{\mathrm{M} 1}$ partition into the interfacial region of DOPC bilayers, about to the level of the glycerol backbone and ester carbonyl groups (26). This is a region that is energetically favorable for small Trpcontaining model peptides (27). It is therefore not surprising that OmpA folding progresses relatively quickly to this state. In contrast, the Trps in $\mathrm{I}_{\mathrm{M} 2}$ have to penetrate more into the hydrocarbon layer, approximately to the region of highest $\mathrm{CH}_{2}$ group density (26) and order (28). Not surprisingly, $k_{2}$ is much slower $\left(28\right.$ times at $20^{\circ} \mathrm{C}$ ) than $k_{1}$. Unfortunately, the experimentally accessible temperature intervals for each step are too narrow to calculate activation energies which would have been interesting to compare and relate to the known bilayer structure in each transition region. However, even without measuring activation energies per se, it is clear from the temperature dependence of our data that the bilayer presents two major energy barriers to the translocation of OmpA. The first "resistance" occurs upon translocation beyond the carbonyl group into the methylene chain region of the cis monolayer, and the second "resistance", which is only overcome at temperatures above $27.5^{\circ} \mathrm{C}$, is presented by the high $\mathrm{CH}_{2}$ packing density of the trans monolayer. A plausible explanation for our result that the trans monolayer imposes a higher energy barrier to translocation than the cis monolayer is the higher lipid packing density in the inner leaflet of the small sonicated unilamellar vesicles that were used in this work. At $27.5^{\circ} \mathrm{C}$, the Trps of the protein are trapped in a metastable state between these two energy barriers. At $40^{\circ} \mathrm{C}$, the second energy barrier is overcome in about $10-20 \mathrm{~min}$, but at $28-30^{\circ} \mathrm{C}$ this step is more complex and is characterized by a biexponential time course.

It is also instructive to observe the Gaussian spread parameter $\sigma$ during OmpA refolding in DOPC (Figure 7). At most temperatures, these widths decrease as a function of time, presumably indicating decreasing heterogeneity (or entropy) in the system as folding proceeds. It is likely that multiple folding pathways coexist in this membrane protein 
as has been postulated for many soluble proteins. The focusing of the spread parameter $\sigma$ as a function of time could therefore be a direct consequence of the narrowing of conformational space as the protein progresses to lower energy states in the "folding funnel". The only exception occurs at $27.5^{\circ} \mathrm{C}$, the metastable state, where the distribution of Trps becomes wider (in fact, as wide as the bilayer itself) as time progresses. This wide distribution may be related to the metastability of this state and/or the fact that only four of the five Trps cross the lipid bilayer according to prediction (5) and experiment (19). The final widths of the fluorescence quenching profiles obtained at all other temperatures are similar to values obtained on membrane proteins and model compounds in thermodynamic equilibrium. For example, a profile width of $\sigma=4.4 \AA$ was calculated for cytochrome $b_{5}$, and similar values were obtained for melittin and tryptophan octyl ester $(22,29)$. This comparison indicates that the majority of the five Trp residues of OmpA are located at similar distances from the bilayer center and that these intermediates are relatively stable over longer times at each temperature. In contrast, at $2{ }^{\circ} \mathrm{C}$ the profile width is 2-4 times broader, which would indicate that the five Trps are located at different distances. These interpretations are confirmed by individually locating the Trps of five single Trp mutants of OmpA at 2 and $40{ }^{\circ} \mathrm{C}(19)$.

We chose to analyze our data by distribution analysis. The major conclusions of this work do not depend on this choice, and we believe we would have obtained similar timedependent Trp trajectories, had we chosen the parallax method which has also been widely used to measure equilibrium depths in lipid bilayers. However, the parallax method requires that the most efficient quenchers be selected in each situation (temperature, time, etc.), which makes the analysis more tedious than distribution analysis. In distribution analysis, all quenchers are fit simultaneously to obtain the pertinent quenching profiles. In addition, the distribution parameter $\sigma$, which does provide interesting new information on the folding mechanism of OmpA, is obtained from a distribution analysis, but not from the parallax method.

It is interesting to compare the acrylamide quenching data (Figure 8) with similar experiments by Rodionova et al. (7). In that earlier work, acrylamide quenching was measured with OmpA that was reacted with DMPC bilayers at 10 and at $30^{\circ} \mathrm{C}$. OmpA was protected from acrylamide quenching at both temperatures. In the present experiments, OmpA was not protected from acrylamide quenching when experiments were performed at $2{ }^{\circ} \mathrm{C}$. The results of both studies are consistent with a shallow Trp location at $2{ }^{\circ} \mathrm{C}$ and a deeper Trp location at $7-26{ }^{\circ} \mathrm{C}$. Since Rodionova et al. (7) investigated partially folded OmpA in DMPC at $10^{\circ} \mathrm{C}$, it must be concluded that OmpA can penetrate the gel phase of this lipid to some extent. Whether or not the $10{ }^{\circ} \mathrm{C}$ intermediates in DMPC and DOPC resemble each other is presently not known.

The results of this paper also offer an explanation for some previously unexplained results that were obtained when OmpA was refolded in DOPC. We previously detected significant amounts of inserted OmpA only at temperatures above $30{ }^{\circ} \mathrm{C}$ (see Figure 1 in ref 2). We also found that unboiled OmpA migrated on SDS gels at an apparent molecular mass of $32 \mathrm{kDa}$ (i.e., between 35 and $30 \mathrm{kDa}$ for surface-adsorbed and completely refolded OmpA, respec- tively) when it was reacted with DOPC at $30^{\circ} \mathrm{C}$ for only a few minutes. However, longer incubation times lead to the final $30 \mathrm{kDa}$ form. We now think that the $32 \mathrm{kDa}$ form most likely represents the $\mathrm{I}_{\mathrm{M} 3}$ intermediate, which is characterized by a Trp location close to the membrane center. These results suggest further that the protein has become significantly more compact at this stage. Therefore, the $\mathrm{I}_{\mathrm{M} 3}$ intermediate appears to resemble the native state quite closely. It may represent the membrane protein analogue of the "molten globular" state that has been described for many soluble proteins. In contrast, the $I_{M 1}$ intermediate is clearly a more surface-located and less compact intermediate which, however, already contains a significant amount of secondary structure; see Rodionova et al. (7). Therefore, this state maybe more appropriately called a "molten disk" state.

In conclusion, we have detected and characterized by timeresolved distance determinations by fluorescence quenching three different membrane-bound folding intermediates of OmpA. The protein becomes progressively more compact and has to overcome at least two different energy barriers as it translocates parts of its sequence across the lipid bilayer. These results were obtained by introducing TDFQ as a new technique to study membrane protein folding and insertion. We expect TDFQ to become a generally applicable method to study the insertion of membrane proteins at a reasonably high spatial resolution along the bilayer normal. The method should be particularly powerful when combined with introducing, by site-directed mutagenesis, single Trp residues into the membrane protein of interest. We have taken this approach in the accompanying paper (19) where we have replaced all but one Trp in OmpA by phenylalanines and permuted the single Trp position to all five wild-type positions of this residue. The results obtained with the single Trp mutants generally support our interpretations of the data of this paper and additionally resolve the projected trajectories of each of the four $\beta$-hairpins across the lipid bilayer.

\section{ACKNOWLEDGMENT}

We thank Dr. Peter Holloway for the kind gift of some of the brominated lipids. We also acknowledge Dennis Rinehart for excellent technical assistance.

\section{REFERENCES}

1. Surrey, T., and Jähnig, F. (1992) Proc. Natl. Acad. Sci. U.S.A. $89,7457-7461$.

2. Kleinschmidt, J. H., and Tamm, L. K. (1996) Biochemistry 35, 12993-13000.

3. Popot, J. L., Gerchman, S. E., and Engelman, D. M. (1987) J. Mol. Biol. 198, 655-676.

4. Hunt, J., Earnest, T. N., Bousché, O., Kalghatgi, K., Reilly, K., Horváth, C., Rothschild, K. J., and Engelman, D. M. (1997) Biochemistry 36, 15156-15176.

5. Vogel, H., and Jähnig, F. (1986) J. Mol. Biol. 190, 191-199.

6. Koebnik, R., and Krämer, L. (1995) J. Mol. Biol. 250, 617626.

7. Rodionova, N. A., Tatulian, S. A., Surrey, T., Jähnig, F., and Tamm, L. K. (1995) Biochemistry 34, 1921-1929.

8. Grigorieff, N., Ceska, T. A., Downing, K. H., Baldwin, J. M., and Henderson, R. (1996) J. Mol. Biol. 259, 393-421.

9. Pebay-Peyroula, E., Rummel, G., Rosenbusch, J. P., and Landau, E. M. (1997) Science 277, 1676-1681.

10. Schweizer, M., Hindennach, M., Garten, W., and Henning, U. (1978) Eur. J. Biochem. 82, 211-217.

11. Dornmair, K., Kiefer, H., and Jähnig, F. (1990) J. Biol. Chem. 265, 18907-18911. 
12. Surrey, T., Schmid, A., and Jähnig, F. (1996) Biochemistry 35, 2283-2288.

13. Kleinschmidt, J. H., Wiener, M. C., and Tamm, L. K. (1999) (submitted for publication).

14. Eftink, M. R. (1991) in Topics in Fluorescence Spectroscopy (Lakowicz, J. R., Ed.) pp 53-126, Plenum Press, New York.

15. London, E., and Feigenson, G. W. (1981) Biochemistry 20, 1932-1938.

16. Markello, T., Zlotnick, A., Everett, J., Tennyson, J., and Holloway, P. W. (1985) Biochemistry 24, 2895-901.

17. McIntosh, T. J., and Holloway, P. W. (1987) Biochemistry $26,1783-1788$.

18. Wiener, M. C., and White, S. H. (1991) Biochemistry 30, 6997-7008.

19. Kleinschmidt, J. H., den Blaauwen, T., Driessen, A. J. M., and Tamm, L. K. (1999) Biochemistry 38, 5006-5016.

20. Chattophdahyay, A., and London, E. (1987) Biochemistry 26 , $39-45$.
21. Ladokhin, A. S., Holloway, P. W., and Kostrzhevska, E. G. (1993) J. Fluorescence, 195-197.

22. Ladokhin, A. S., and Holloway, P. W. (1995) Biophys. J. 69, 506-517.

23. Abrams, F. S., and London, E. (1993) Biochemistry 32, 10826-10831.

24. Ladokhin, A. S. (1997) Methods Enzymol. 278, 462-473.

25. Wimley, W. C., and White, S. H. (1998) Biophys. J. 74, A15.

26. Wiener, M. C., and White, S. H. (1992) Biophys. J. 61, 437447.

27. Jacobs, R. E., and White, S. H. (1989) Biochemistry 28, 34213437.

28. Seelig, J., and Seelig, A. (1980) Q. Rev. Biophys. 13, 19-61.

29. Ladhokin, A. S., Wang, L., Steggles, A. W., and Holloway, P. W. (1991) Biochemistry 30, 10200-10206.

BI9824644 